\title{
Magnetic Interference on the Infrastructure for a Super-speed Tube Train
}

\author{
Hyung-Woo Lee ${ }^{\dagger}$, Seung Yup Jang*, Bu-Byoung Kang*, Su-Yeon Cho** and Ju Lee**
}

\begin{abstract}
The super-speed tube train was introduced to increase the speed of ground transportation. It levitates magnetically and runs in a partial vacuum tube, which significantly reduces air resistance. However, strong magnetic force sufficient to propel the massive train can affect the infrastructure. The induced eddy current produces joule heat, and raises the inside temperature of the girder, which might lead to electrochemical corrosion on the girder, thereby weakening its durability. In this paper, the authors analyzed the magnetic flux and induced eddy current in the reinforced concrete girder by using three-dimensional FEM, particularly by varying the number of reinforcing steels of the upper flange of the girder to the condition of almost the same flexural strength and reinforcing steel amount.
\end{abstract}

Keywords: Eddy current, Maglev train, Super-speed tube train, Tube infrastructure

\section{Introduction}

The demand for high-speed transportation has recently increased along with globalization. Speed is one of the key parameters for competitiveness especially in Northeast Asia, which will become a world economic center in the near future. New-generation, high-speed transportation systems must have certain requisites, such as rapidity, reliability, and safety. These systems should also be environment-friendly, convenient, low maintenance, compact, lightweight, and suitable for mass-transportation. The Magnetic levitation (Maglev) train is one of the best examples that satisfy these requirements [1]. It replaces wheels by using electromagnets and levitates on the guideway, producing propulsion force electromagnetically without any contact.

The Maglev train dates back to 1934, when Hermann Kemper of Germany patented it. Since then, the development of the Maglev train went through a quickening period of the 1960s, the maturity of the 1970s-1980s, and the test period of the $1990 \mathrm{~s}$, finally accomplishing practical public service in 2003 in Shanghai, China [2]-[5]. The Maglev train offers numerous advantages over the conventional wheel-on-rail system; thus, global transportation is advancing toward the Maglev train.

However, although the high-speed Maglev train has many advantages and is a promising piece of machinery, there is a critical speed limitation of around $500(\mathrm{~km} / \mathrm{h})$. As

\footnotetext{
$\dagger$ Corresponding author: Dept. of Vehicle Dynamics \& Propulsion System Research, Korea Railroad Research Institute, Korea. (krhwlee@krri.re.kr)

* Dept. of Vehicle Dynamics \& Propulsion System Research, Korea Railroad Research Institute, Korea

** Dept. of Electrical Engineering, Hanyang University, Korea. (julee@hanyang.ac.kr).

Received: June 23, 2010; Accepted: January 18, 2011
}

the speed of the train increases, it produces severe aerodynamic noise and air resistance, which increase energy consumption by geometric progression. The aerodynamic noise is six times louder, the air resistance is quadratically proportional to the velocity, and the required power is proportional to the third power of the train velocity. Therefore, air resistance becomes dominant in a high-speed operation, with over $85(\%)$ of total running resistance at the speed of $500(\mathrm{~km} / \mathrm{h})$, as shown in Fig. 1.

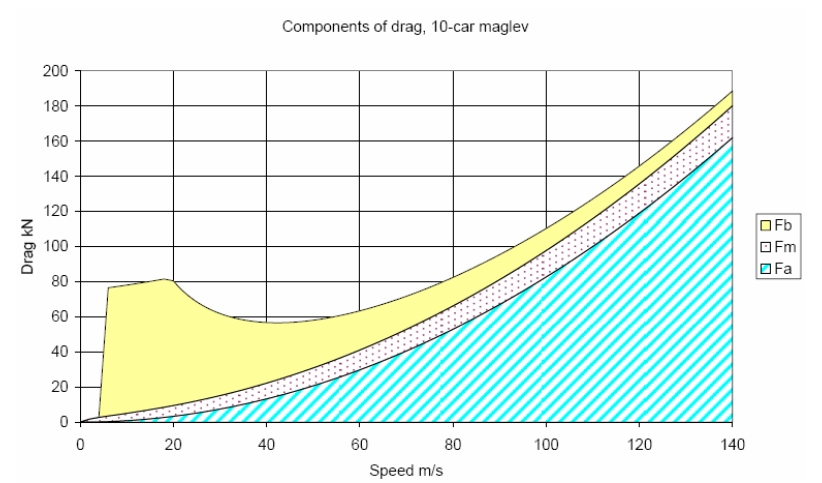

$F_{b}$ : Drag created by the electrical system producing auxiliary power

$\mathrm{F}_{\mathrm{m}}$ : Magnetic drag

$\mathrm{F}_{\mathrm{a}}:$ Aerodynamic drag $(1[\mathrm{~m} / \mathrm{s}]=3.6[\mathrm{~km} / \mathrm{h}])$

Fig. 1. Components of train drag [6]

In order to solve these problems, the concept of the tube train has been proposed in Switzerland and Korea [7], [8]. Whereas conventional Maglev trains run in open space of 1 (atm), the super-speed tube train levitates magnetically and runs in a partial vacuum tube of around 0.1 (atm), which can reduce the air resistance significantly. 


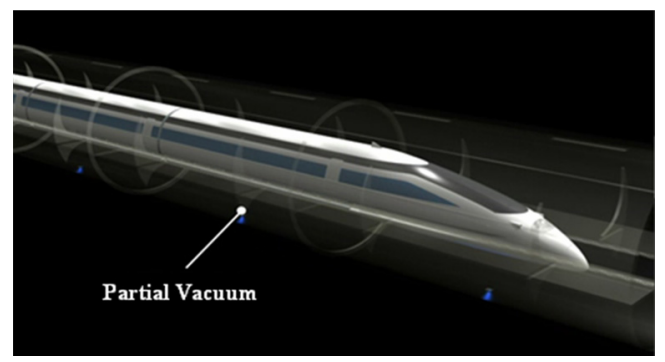

Fig. 2. Conceptual drawing of the tube train [8]

The infrastructure of the super-speed tube train is divided to "girder" and "tube." Girder is generally made of reinforced concrete to support the moving load, and tube is made of cheap cement concrete to reduce the construction cost, even though the air tightness of the cement concrete is inferior to the iron and aluminum tubes.

In a previous work, the magnetic effect on the tube has been analyzed according to the materials and diameters of the tube [9]. Results showed that cement concrete tubes have minor magnetic effect, and the girder of reinforced concrete, which has steel in it, may cause worse problems.

In general, traveling magnetic field generated from the stator winding of the propulsion system becomes linked with the moving electromagnets installed in the vehicle. However, the magnetically linked section [approximately $0.1(\mathrm{~km})]$ is only approximately $10(\%)$ of the energized section [around $1.2(\mathrm{~km})$ ] and leakage flux penetrates to the infrastructure at the nonlinked section [90 (\%) of the energized section at around $1.1(\mathrm{~km})]$. This time-varying magnetic field induces the electromotive force on the tube and girder, and generates the eddy current on the reinforced bar (conductor) inside of the girder. This eddy current can produce joule heat and raise the temperature inside the girder, thereby cracking it. In addition, this current can lead to electrochemical corrosion and reduce its durability.

In this paper, the authors have analyzed the leakage flux and induced eddy current on the reinforced concrete girder by using three-dimensional finite element method (FEM). These effects are investigated by varying the number of reinforcing steels of the upper flange of the girder to the condition of almost same flexural strength, and reinforcing steel amount in order to hold the construction cost.

\section{Analysis Model}

As the magnetically linked section does not cause any problem, only the nonlinked section is considered in the research. Fig. 3 shows the analysis model. Reference dimension of the model is as follows: tube diameter is $3(\mathrm{~m})$; girder width is 1,280 and $800(\mathrm{~mm})$, respectively; and rail height is $878(\mathrm{~mm})$. Specification of the analysis model is represented in Table 1. The data of the current magnitude and pole pitch are brought from the German Maglev train, TR06. The frequency is $378(\mathrm{~Hz})$ because the velocity of the train is approximately $700(\mathrm{~km} / \mathrm{h})$ for super-speed tube train. The reference of the tube diameter is $3,000(\mathrm{~mm})$, which is temporarily designated for super-speed tube trains.

The induced eddy currents are analyzed in four cases of reinforcing steel of the upper flange: $6,8,12$ and 20 (ea). The reinforcing steel must provide nearly the same flexural strength and consume practically the same amount of steel for comparison.

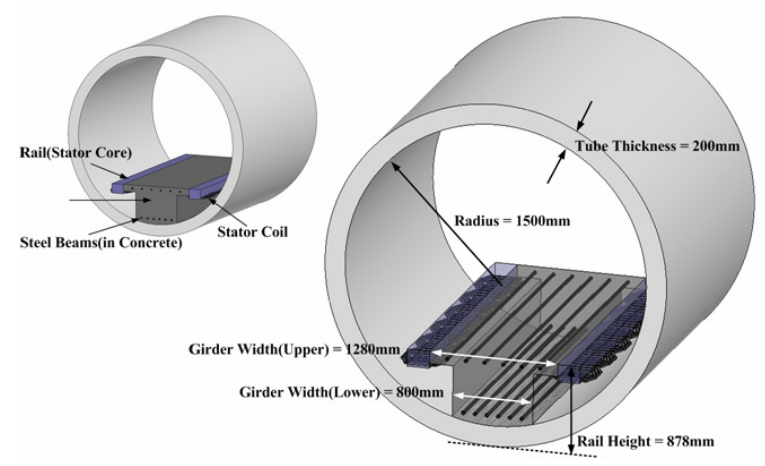

Fig. 3. Analysis model of the tube infrastructure

Table 1. Specification of the model

\begin{tabular}{c|c}
\hline Variable & Value \\
\hline \# of turns of the stator windings & $1($ turn $)$ \\
\hline Current magnitude & $1,200(\mathrm{~A}$, peak $)$ \\
\hline Frequency & $378(\mathrm{~Hz})$ \\
\hline Pole pitch & $258(\mathrm{~mm})$ \\
\hline Velocity & $702(\mathrm{~km} / \mathrm{h})$ \\
\hline Thickness of the tube & $200(\mathrm{~mm})$ \\
\hline Diameter of the tube & $3,000(\mathrm{~mm})$ \\
\hline
\end{tabular}

Fig. 4 shows two of the sample cases. The diameter of the reinforcing steel of the upper flange of each case is different in order to maintain the same amount of steel. In contrast, lower beams are the same at 6 (ea) because they do not have an influence on the leakage flux in staying away from the stator windings.

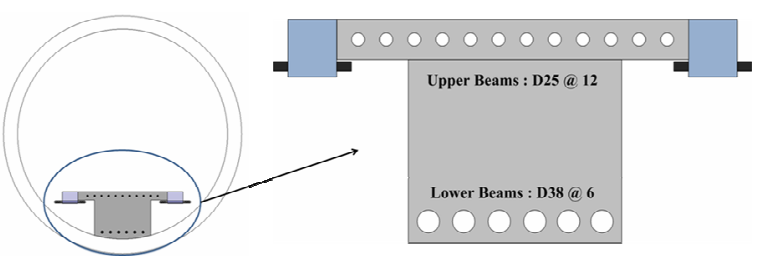

(a) Case of 12 reinforcing steel

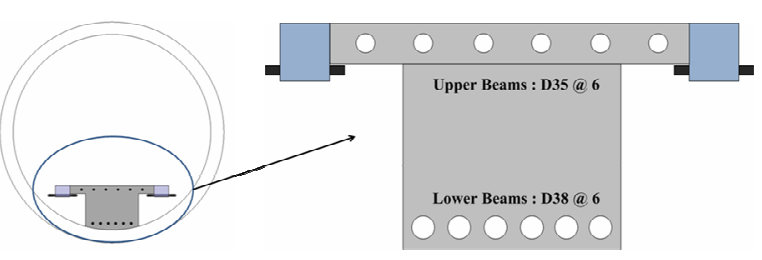

(b) Case of 6 reinforcing steels

Fig. 4. Analysis cases of reinforcing steels of the upper flange 


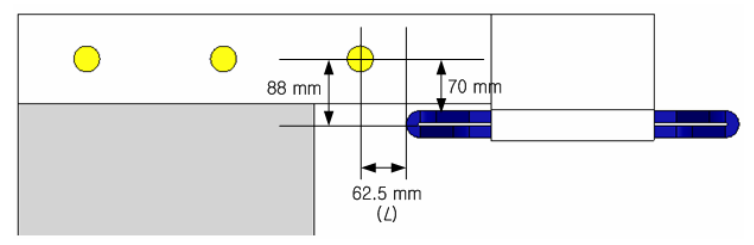

(a) Case of 6 reinforcing steel

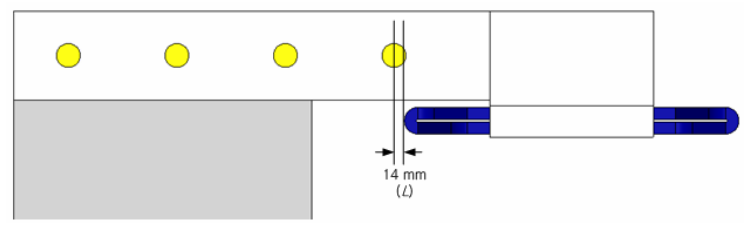

(b) Case of 8 reinforcing steel

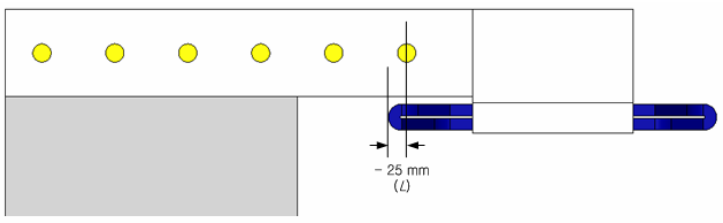

(c) Case of 12 reinforcing steel

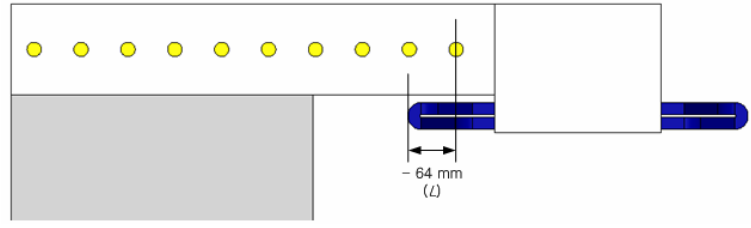

(d) Case of 20 reinforcing steel

Fig. 5. Relative distance $(L)$ of the reinforcing steel of the upper flange in the analysis cases

Fig. 5 shows each distance $L$ of the reinforcing steels of the upper flange in the analysis cases.

The relative distance $L$ is between the center of the outmost reinforcing bar of the flange and the outline of the end turn of the propulsion coil. By definition, the relative distance, $L$, will be zero, and as the number of reinforcing steel increases, $L$ becomes a negative value.

\section{FEM Analysis}

The shape of the analysis model makes two-dimensional (2-D) analysis impossible; therefore, three-dimensional (3D) analysis is necessary. Considering the symmetry of the propulsion coil, the half model is used to save analysis time and computer memory. Using 3-D analysis, the normal component of the magnetic flux density has been analyzed because the eddy current is generated by the normal vector of the magnetic flux density on the surface.

Fig. 6 shows the 3-D half model with the symmetric boundary condition (a), mesh model (b), and magnetic flux density vector distribution through analysis model (c).

The amount of eddy current is proportional to the velocity, conductivity, and flux density. Because the conductivity of the steel is constant at $10.3 \times 10^{6} \mathrm{~S} / \mathrm{m}$, and the velocity is constant at $700(\mathrm{~km} / \mathrm{h})$ [operating frequency: $378(\mathrm{~Hz})$ ], the eddy current is dependent on the flux density distribution. Therefore, the peak value of the eddy current will be different in each case $[6,8,12,20$ (ea) of reinforcing steel].

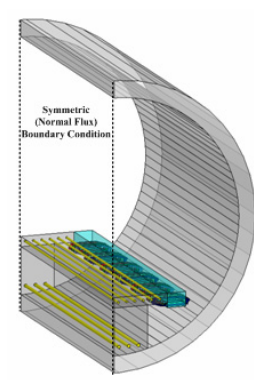

(a)

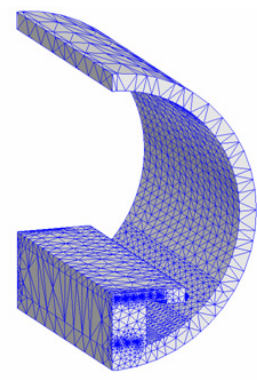

(b)

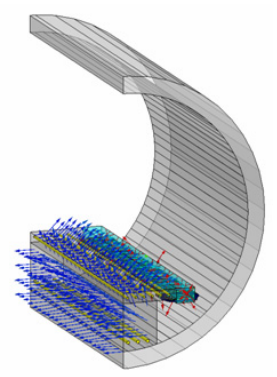

(c)
Fig. 6. 3-D half analysis model

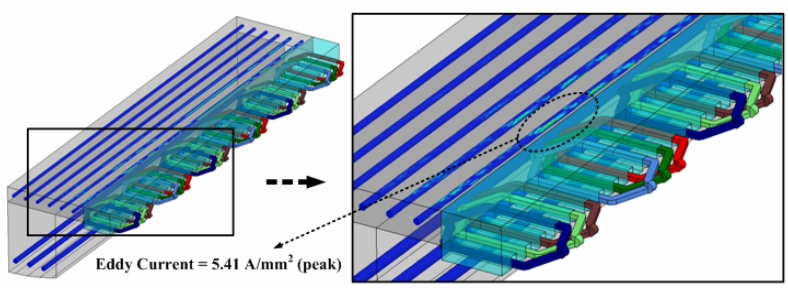

Fig. 7. Eddy currents on the reinforcing steel of the upper flange [12 (ea)]

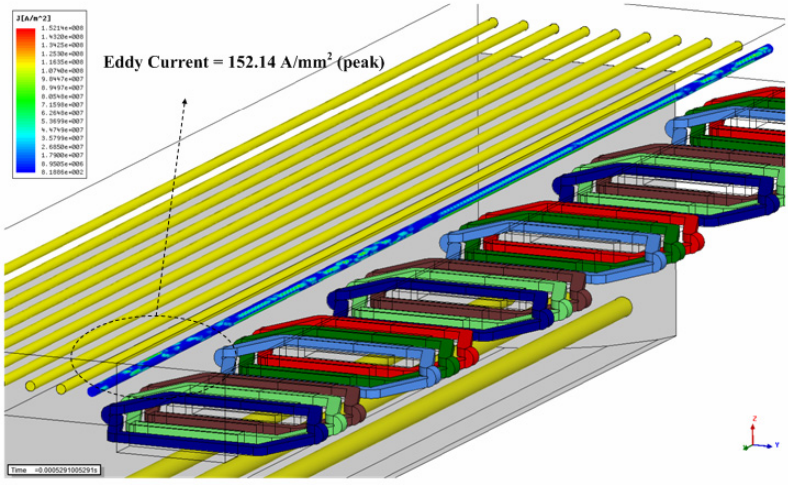

Fig. 8. Eddy currents on the reinforcing steel of the upper flange [20 (ea)]

Fig. 7 shows the eddy currents on the reinforcing steel of the upper flange in the case of 12 (ea). The closer reinforcing steels locate to the propulsion coil, the more eddy currents are induced. The instantaneous peak magnitude of the eddy current density is $5.41 \mathrm{~A} / \mathrm{mm}^{2}$.

In the case of 20 (ea), the exterior reinforcing steel is closer to the propulsion compared to the case of 12 (ea). 
Therefore, much more eddy current $\left(152.14 \mathrm{~A} / \mathrm{mm}^{2}\right)$ is induced. This periodic eddy current produces joule heat and raises the temperature of the reinforcing bars in very short period of time. This instant elevated temperature of the reinforcing steel causes the thermal strain on the surrounding concrete, which can consequently induce cracks on it.

Table 2 shows the peak eddy current density for each case. From the analysis, the peak value of the eddy current density increased sharply passing a certain distance. There is a critical relative distance from the magnetic point of view, between $-25(\mathrm{~mm})$ and $-64(\mathrm{~mm})$. This implies that, for the construction of the guideway structure, the minimum distance between the reinforcing bar and the propulsion coil should be specified to keep the eddy current below a certain allowable value.

Table 2. The peak eddy current density on the reinforcing steel of the upper flange according to the distance between reinforcing bar and the propulsion coil

\begin{tabular}{c|c}
\hline Variable & Value \\
\hline \# of turns of the stator windings & $1($ turn $)$ \\
\hline Current magnitude & $1,200(\mathrm{~A}$, peak $)$ \\
\hline Frequency & $378(\mathrm{~Hz})$ \\
\hline Pole pitch & $258(\mathrm{~mm})$ \\
\hline Velocity & $702(\mathrm{~km} / \mathrm{h})$ \\
\hline Thickness of the tube & $200(\mathrm{~mm})$ \\
\hline Diameter of the tube & $3,000(\mathrm{~mm})$ \\
\hline
\end{tabular}

In Table 2, the diameter of the reinforcing steel of the upper flange for each case is also different. Therefore, the skin effect, due to various diameters, must be considered because it may modify the analysis. Skin effect is the tendency of an alternating electric current (AC) to distribute itself within a conductor so that the current density near the surface of the conductor is greater than that at its core, while skin depth is the average depth of the skin. The skin depth can be calculated using Formula (1):

$$
\delta=\sqrt{\frac{2 \rho}{\omega \mu}}
$$

where $\rho$ is resistivity, $\omega$ is angular frequency, and $\mu$ is magnetic permeability. The skin depth of the reinforcing steel in the paper is as follows:

$$
\begin{aligned}
\delta & =\sqrt{\frac{2 \rho}{\omega \mu}=\sqrt{\frac{\frac{2}{1.03 \times 10^{7}}}{2 \pi \times f \times \mu_{0} \mu_{r}}}} \\
& =\sqrt{\frac{2}{2 \pi \times 378 \times 4 \pi \times 10^{-7} \times 5000}}=0.114[\mathrm{~mm}]
\end{aligned}
$$

The smallest diameter of the reinforcing steel of the up- per flange is $19(\mathrm{~mm})$ and largest is $35(\mathrm{~mm})$. Therefore, the skin depth is much smaller than the smallest diameter of the reinforcing steel of the upper flange; the skin depth does not have a major effect on the different reinforcing steels and the distance $L$ is significant.

\section{Conclusion}

By reducing the aerodynamic noise and air resistance to increase the speed of ground transportation, the superspeed tube train, which levitates magnetically and runs in a partial vacuum tube, has been introduced. In this system, magnetic interference with the tube infrastructure cannot be overlooked because the magnetic energy is sufficiently strong to propel the massive train.

The magnetic flux and induced eddy current on the reinforcing steels of the girder have been investigated by 3-D FEM. From the simulation results, a critical distance between the reinforcing bar and the propulsion coil can induce a large amount of the eddy current that produces joule heat and causes the thermal strain inside the girder that may lead to electro-chemical corrosion and, consequently, reduce the durability. For construction, this should be considered carefully.

\section{References}

[1] H.-W. Lee, K.-C. Kim, and J. Lee, "Review of Maglev train technologies," IEEE Trans. Magnetics, vol. 42, pp. 1917-1925, Jul. 2006.

[2] Yamamura S., "Magnetic levitation technology of tracked vehicles present status and prospects," IEEE Trans. Magnetics, vol. 12, pp. 874-878, Nov. 1976.

[3] Sinha P., "Design of a magnetically levitated vehicle,” IEEE Trans. Magnetics, vol. 20, pp. 1672-1674, Sep. 1984.

[4] Rogg D., "General survey of the possible applications and development tendencies of magnetic levitation technology," IEEE Trans. Magnetics, vol. 20, pp. 1696-1701, Sep. 1984.

[5] Eastham A.R., Hayes W.F., "Maglev systems development status," IEEE Aerospace and Electronic Systems Magazine, vol. 3, pp. 21-30, Jan. 1988.

[6] R. Kemp and R Smith, "Technical issues raised by the proposal to introduce a $500 \mathrm{~km} / \mathrm{h}$ magnetically levitated transport system in the UK," Report on Imperial College London and Lancaster University, pp. 12, 2007.

[7] M. Mossi and P. Rossel, "Swissmetro : a revolution in the high-speed passenger transport systems," in 2001 Swiss Transport Research Conference, pp. 1-16.

[8] B. Kang, et al., "Development of the core technologies for the ultra high-speed tube train," Korea Rail- 
road Research Institute report, Dec. 2009.

[9] H.-W. Lee, S.-Y. Cho, W. Cho, J. Lee and H.-B. Kwon, "Analysis of the magnetic effect on the tube infrastructure for a super-speed tube train," International Journal of Railway, vol. 2, pp. 170-174, Dec. 2009.

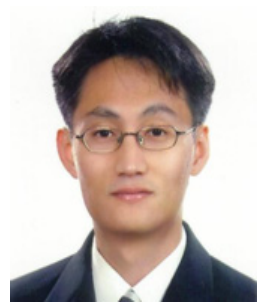

Hyung-Woo Lee received his Electrical Engineering B.S. and M.S. degrees from Hanyang University, Seoul, Korea, in 1998 and 2000, respectively, and the Ph.D. degree from Texas A\&M University, College Station, TX, in 2003. In 2004, he was a Post-doctoral Research Assistant in the Department of Theoretical and Applied Mechanics, Cornell University, Ithaca, NY. In 2005, he was a contract Professor at the BK division of Hanyang University, Seoul, Korea. Since 2006, he has been a Senior Researcher at the Korea Railroad Research Institute, Uiwang, Korea. His research interests include design, analysis and control of motor/generator, power conversion systems, applications of motor drives such as Maglev trains, robots, and modern renewable energy systems.

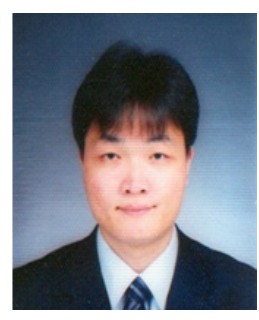

Seung Yup Jang received B.S., M.S. and $\mathrm{Ph} . \mathrm{D}$. degree in civil engineering from Seoul National University in 1995, 1997 and 2003, respectively. He has been a senior researcher at Korea Railroad Research Institute, Uiwang, Korea since 2003. His research interests include concrete slab track and concrete materials science.

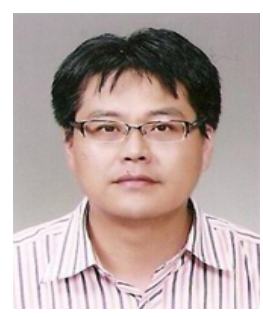

Bu-Byoung Kang was born in Korea in 1969. He received his B.Sc. and Master degrees in Mechanical Engineering from the Korea University in 1992 and 1996, respectively. He received a Ph.D. degree in Mechanical Engineering from Imperial College London in 2008. He worked in Korea High Speed Rail Construction Authority from 1996-1997. He then joined Korea Railroad Research Institute, rolling stock division first as a researcher, then senior researcher. He has worked on a wide variety of projects about highspeed train, wheel-rail interface, test and evaluation of railway vehicle, railway safety research, research and development in system interface between railway vehicle and infrastructure. He has worked in super-speed train research department since 2008.

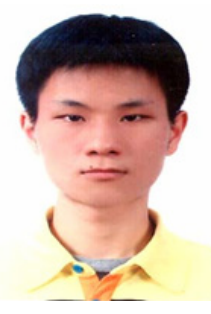

Su-Yeon Cho received his B.S. and M.S. degrees in Electrical Engineering from Han-yang University, Seoul, Korea in 2008 and 2010, respectively. Since 2010, he has been pursuing the Ph.D. degree at the Department of Electrical Engineering, Hanyang University. His research interests include design, analysis, testing and control of motor/generator; power conversion systems; and applications of motor drive, such as electric vehicles, high-speed Maglev train and renewable energy systems.

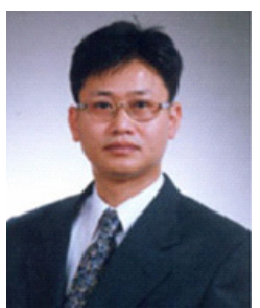

Ju Lee obtained his B.S. and M.S. degrees from Hanyang University, Seoul, Korea in 1986 and 1988, respectively. He obtained his Ph.D. from Kyusyu University, Fukuoka, Japan, in 1997. He worked as an Assistant Researcher at the Agency for Defense Development from 1989 to 1993 and with the Korea Railroad Research Institute in 1997 as Chief of the Division on Light Subway Systems. He joined Hanyang University as Professor of the Department of Electrical Engineering in September 1997. His main research interests include electrical machinery and drives; electromagnetic field analysis; new transformation systems, such as hybrid electric vehicles and high-speed electric trains; and standardization. He is also the General Manager of the Human Resource Development Center for Electric Machine and Devices, Seoul, Korea. 